\title{
Leukocytes and drug-resistant cancer cells are targets for intracellular delivery by
}

\section{adenoviral dodecahedron}

Marta Jedynak ${ }^{1}$, David Laurin, $\mathrm{PhD}^{2}$, Patryk Dolega ${ }^{1}$, Malgorzata Podsiadla-Bialoskorska ${ }^{1}$, Inga Szurgot, $\mathrm{PhD}^{1,3}$, Jadwiga Chroboczek, $\mathrm{PhD}^{2,1}$, Ewa Szolajska, $\mathrm{PhD}^{1^{*}}$

${ }^{1}$ Institute of Biochemistry and Biophysics, Polish Academy of Sciences, Warsaw, Poland;

${ }^{2}$ Université Grenoble Alpes, CNRS, Grenoble INP, TIMC-IMAG, EFS, F38000 Grenoble, France

${ }^{3}$ Current address: Department of Microbiology, Tumor and Cell Biology, Karolinska Institutet, Stockholm, Sweden

*Corresponding author: E.S

E-Mail: ewasz@ibb.waw.pl

Phone: +48 225922420

Abbreviations: Ad Dd, adenoviral dodecahedron; Ad3, adenovirus type 3; BBB, blood-brain barrier; DOX, doxorubicin; FBS, fetal bovine serum; FSC, forward scatter; HCC, hepatocellular carcinoma; HSPGs, heparan sulphate proteoglycans; IC $_{50}$, half maximal inhibitory concentration; i.t., intratumoral; i.v., intravenous; MDR, multidrug resistance; MFI, mean fluorescent intensity; MTT, 3-(4,5-dimethylthiazol-2-yl)-2,5-diphenyltetrazolium bromide; CNS, central nervous system; Pb, penton base; P-gp 1, P-glycoprotein 1; p.i., post injection; $\mathrm{p} / \mathrm{sec} / \mathrm{cm}^{2}$, number of photons per second, per square centimeter; RBC, red blood cells; SSC, side scatter; WBC, white blood cells; VLPs, virus-like particles

ABSTRACT WORD COUNT: 149

TEXT WORD COUNT: 4972

NUMBER OF REFERENCES: 42

NUMBER OF FIGURES: 8

NUMBER OF TABLES: 1

NUMBER OF SUPPLEMENTARY FILES: 1

Funding: This work was supported by the National Science Centre (Poland) decision DEC2013/09/B/NZ3/02327 and in part by Association Espoir Isere Contre Le Cancer and by association Gefluc Grenoble Dauphiné-Savoie.

The authors declare no competing financial interests 


\begin{abstract}
One of the major factors limiting the effectiveness of cancer chemotherapy is inefficient drug delivery. Systems enabling efficient delivery and enhanced intracellular uptake, appear particularly promising in this respect. Virus-like particle, adenoviral dodecahedron (Dd), employs receptor-mediated endocytosis for cell penetration and is able to deliver intracellularly dozens of cargo molecules attached to one particle. We focused on studying Dd properties in the context of cancer treatment, showing that intratumoral injection of Dd, assessed in mouse xenograft model, results in vector accumulation in tumor without spreading in off-target organs. Furthermore, we demonstrated that $\mathrm{Dd}$ is a promising vector targeting leukocytes and drug-resistant cancer cells. Dd uptake by human blood cells analyzed in vitro indicated the preference for leukocytes in comparison to red blood cells and platelets. Furthermore, internalization of Dd-doxorubicin conjugate by drug-resistant cells leads to increased nuclear accumulation of doxorubicin and significant enhancement of cytotoxicity against target cancer cells.
\end{abstract}

Keywords: Adenoviral dodecahedron; virus-like particle; drug delivery, drug resistance, in vivo imaging 


\section{Background}

The main limitation of cancer chemotheraphy is that anticancer agents are nonselective and often toxic for healthy cells. Moreover cancer cells develop resistance to chemotherapy that could not be overcome by enhancement of drug dose, because the amount of chemotherapeutic that can be administered is restricted by its side effects such as cardiotoxicity in case of anthracyclines. The improvement of cancer treatment efficacy and reduction of detrimental side effects to normal tissues may be achieved through the application of drug loaded nano-delivery systems. Currently, there are a number of nanoparticle therapeutics, that have been approved for clinical use, either by the Food and Drug Administration (FDA) in the United States, or the European Medicines Agency (EMA) in the European Union. Majority of them are liposomal systems encapsulating an anticancer drug, such as polyethylene glycol (PEG) functionalized liposomal doxorubicin, that was the first cancer nanomedicine approved by FDA in 1995, or liposomal daunorubicin approved by FDA in 1996. Also nonliposomal preparations such as albumin-bound paclitaxel and polymeric particles, such as methoxy-PEG-poly[DL-lactide]taxol, were launched. All approved delivery systems target tumors passively via the enhanced permeability and retention effect (EPR). ${ }^{1}$ These formulations are less toxic than conventional therapies but are still associated with adverse effects such as stomatitis and palmar-plantar erythrodysesthesia for PEGyleted liposomal $\mathrm{DOX}^{2}$ and sensory neuropathy and nausea for albumin bound paclitaxel. ${ }^{3}$ Thus, there is a need for development of new safe drug nano-delivery systems with improved biodistribution, pharmacokinetic properties and active targeting.

Virus-like particles (VLPs) are an attractive alternative to chemically synthesized nanoplatforms in development of novel carriers for targeted drug delivery, as they do not demonstrate limitations such as toxicity of metal particles, low stability of liposomes nor structural heterogeneity of polymer-based nanoparticles. ${ }^{4}$ Adenoviral dodecahedron (Ad Dd), 
the symmetrical, small $(28 \mathrm{~nm})$ and non-infectious VLP, endowed with extraordinary ability for intracellular penetration, is promising vector-candidate for therapeutic development. The particle is composed exclusively of 12 copies of the pentameric viral protein, adenovirus type 3 (Ad3) penton base $(\mathrm{Pb})$, one of two capsid proteins responsible for virus cell entry. Dd retains usage of receptors, $\alpha v \beta 3$ and $\alpha v \beta 5$ integrins, recognized by $\mathrm{Pb}$ within Ad particle. ${ }^{5} \mathrm{In}$ addition, Dd recognizes heparan sulphate proteoglycans (HSPGs) that do not serve as receptors for Ad3 ${ }^{6,7}$ Importantly, integrins $\alpha v \beta 3$ are abundantly expressed on angiogenic endothelial cells and some tumor cells but not on resting endothelial cells of most normal organs. ${ }^{8,9}$ The presence of 60 RGD motifs responsible for the interaction with $\alpha v$ integrins, suggests Dd affinity to cancer cells. Therefore, our current work is focused on investigation of Dd properties in the context of application in cancer treatment. Dd employs the mechanism of receptor-mediated endocytosis for cell entry and has the ability to escape endosomes before reaching lysosomes, which results in cytoplasmic delivery of cargo molecules in a functional form. ${ }^{10-12}$ The chemical and genetic modifications allow for covalent attachment of cargo molecules or their insertion into particle structure without disrupting VLP integrity or penetration properties. ${ }^{13-15}$ The usefulness of Dd as a carrier for conjugated small molecules was already proven by targeted delivery of anticancer agents to hepatocellular carcinoma (HCC) tumor in animal model, leading to inhibition of tumor growth. ${ }^{14}$ The main obstacle associated with the use of VLPs is potential immunogenicity and toxicity, however, our previously published data indicated slow build-up of the humoral response against Dd as well as the lack of acute toxicity of the vector upon repetitive administration of Dd conjugates. ${ }^{14}$

In the present work we analyzed delivery of doxorubicin (DOX) conjugated to Dd (Dd-DOX) in comparison to free DOX. It was assessed in multidrug-resistant (MDR) human uterine sarcoma MES-SA Dx5 cells, for which we confirmed the expression of $\alpha v \beta 3$ and $\alpha v \beta 5$ integrins involved in Dd internalization. The results of cytotoxicity tests attested the ability of 
Dd for circumvention of the P-glycoprotein 1 (P-gp 1) -mediated multidrug resistance mechanism. We demonstrated that efficient uptake of Dd-DOX conjugate in MES-SA Dx5 cells leads to accumulation of DOX in cell nucleus and significantly enhances drug cytotoxicity in target cells. Since the knowledge of carrier tropism is essential for precise therapeutic application, we investigated two routes of Dd administration, intravenous and intratumoral. This study was performed in mouse xenograft model using in vivo fluorescence imaging. Based on the results, concerning vector accumulation in tumor and tumor-draining lymph nodes without spreading in off-target organs after intratumoral injection, we consider local application as the optimal route of administration. Furthermore, we demonstrated the accumulation of Dd in animal skin after intravenous administration which is consistent with results showing efficient penetration of primary dermal fibroblasts in vitro. The analysis of ex vivo interactions of $\mathrm{Dd}$ with human blood cells, provided information on preferential leukocytes targeting. Additionally, this paper contains a description of lyophilization methodology for long-term storage of the VLP.

\section{Methods}

Section containing methods concerning:

Materials

Cell lines and culture media

Vector preparation

Dd labeling

Dd imaging in mice xenograft model

Dd uptake by human dermal fibroblasts

Dd uptake by human blood cells

Detection of $\alpha v \beta 3$ and $\alpha v \beta 5$ integrins in human sarcoma cells

Cellular uptake of Dd in drug resistant cells

Corrected total cell fluorescence measurement

Conjugation of doxorubicin to dodecahedron particles 


\section{Cellular uptake of Dd-DOX conjugate}

MTT cell viability assay

\section{Dd lyophilization}

\section{Electron microscopy analysis}

is placed in Supplemental materials.

\section{Results}

\section{In vivo Dd-AF680 distribution}

We applied non-invasive fluorescence tomography in xenograft model of mice bearing subcutaneous melanoma tumor for identification of the best route of Dd administration for optimal tumor accumulation and limited non-specific toxicity of anticancer agents delivered by VLP. We monitored tumor uptake of Dd labeled with Alexa Fluor 680 (Dd-AF680), either after intravenous (i.v.) application, which is the most common method of chemotherapy administration or after intratumoral (i.t.) injection. In vivo imaging after i.v. administration revealed the presence of uniform fluorescence signal across mice body visible $5 \mathrm{~h}$ p.i, which disappeared almost completely after 24 h p.i. (Figure 1, A). The uniform signal masked the fluorescence from tumor (5 h p.i.), which was revealed by ex vivo fluorescence measurements (Figure 1, B and $C$ ). Intravenous injection resulted in a spread distribution in off-target organs. Ex vivo imaging at 5 h p.i. demonstrated the strongest signal in skin and liver, and a weak signal in tumor and organs such as bladder, spleen, kidneys, adrenal, fat, lungs, uterus and guts (and a nonspecific auto-fluorescence in the stomach) (Figure 1, $B$ and $C$ ). Dd-AF680 was also detected in isolated sciatica-, inguinal- and axillary lymph nodes (Figure $\mathrm{S} 1, A$ ), which indicates that Dd entered into lymphatics after extravasation from the vascular system to interstitial areas of several organs. Within $24 \mathrm{~h}$ the particle has been eliminated from all organs except liver, displaying weak fluorescent signal. Dd accumulation in liver upon systemic application was expected, since it is the usual clearance pathway for nanoparticles, in which the important role plays the uptake by the endothelial cells of liver sinusoids. ${ }^{16}$ 
However, the uptake by the liver did not compromise Dd distribution throughout the mice body, which resulted in the preferential accumulation of VLP in animal skin. We demonstrated only limited Dd localization and retention in mice melanoma tumors upon intravenous injection (Figure 1), therefore we further analyzed the effect of direct intratumoral application on the distribution of VLP. Tracking of Dd upon i.t. injection demonstrated accumulation of Dd-AF680 in tumor at 5 and 24 h p.i. (Figure 2, A). Ex vivo imaging and analysis of isolated organs confirmed persistence of Dd-AF680 in tumors at 5 and 24 h p.i., without localization in off-target organs, except residual signal in liver and skin

(Figure 2, $B$ and $C$ ). Importantly, the particle was present for $24 \mathrm{~h}$ p.i. in tumor-draining lymph nodes, being the first site of metastasis in most types of cancer (Figure S1, B). Of note, it appears that Dd is unable to pass the blood-brain barrier (BBB), as no signal was observed upon ex vivo imaging of isolated brains (Figure 1, B and 2, B). It is consistent with our results of in vitro studies, in which either co-culture endothelial BBB model or MDCKII-MDR1 BBB surrogate model were used (results not shown).

\section{Dd uptake by human dermal fibroblasts}

The results of in vivo imaging after intravenous injection of Dd showed particle accumulation in animal skin (Figure 1, $B$ and $C$ ). Therefore, we investigated the ability of Dd to penetrate dermal fibroblasts, the main cells of the skin dermis. Our results confirmed that Dd applied to human primary dermal fibroblasts, easily penetrates them. Internalization of Dd-AF647 increases with the rising Dd concentrations reaching over $90 \%$ and almost $100 \%$ of transduced cells for 10 and $30 \mu \mathrm{g} / \mathrm{ml} \mathrm{Dd}$, respectively (Figure 3).

\section{Dd uptake by human blood cells}

Targeting ability of Dd toward human blood cells is important factor for its application as drug carrier. Therefore fresh total blood was evaluated for transduction by Dd labeled with Alexa Fluor 647 fluorescent dye (Dd-AF647), to analyze target cell types in a single tube by 
flow cytometry. When blood samples were treated for $1 \mathrm{~h}$ with Dd-AF647 (range 0.7 - 12.2 pmoles) affinity to red blood cells and platelets was quite low in comparison to leukocytes (Figure 4, $A$ and $C$ ). Indeed, percent of transduced cells were similar at 0.7 and 1.4 pmoles of Dd but the mean fluorescent intensity (MFI) was high in leukocytes when compared to red blood cells and platelets. When exposed to higher concentrations of $\mathrm{Dd}$, the percent of transduced cells increased in all cells, but with a superior efficiency on leukocytes, close to $50 \%$ platelets and $\mathrm{RBC}$ were transduced at the maximum, whereas it reached $85 \%$ on leukocytes. More notably, at 12.2 pmoles Dd treatment of blood, leukocyte MFI reached 45 times the values observed for $\mathrm{RBC}$ and platelets (Figure $4, A$ and $C$ ). Since the MFI is proportional to the number of fluorescent molecules, we can conclude that Dd preferentially penetrates leukocytes. We also analysed how the Dd transduction depends on leukocytes subtypes (Figure 4, B). We focused on T-cells since they are lymphoid cells and are easily identified by the specific expression of CD3 from their receptor. We also explored Dd transduction on cells specialized into foreign molecules capture. We therefore analyzed $\mathrm{CD}_{3}{ }^{+}$cells since this protein is expressed on membrane of myeloid lineage cells (including polynuclear cells, phagocytes and subtypes of dendritic cells) and CD14 that is expressed, in blood, mainly but not exclusively, by monocytes. All leukocytes were efficiently transduced by Dd and no distinctive amount of fluorescent dye was detected on different leukocytes subtypes (Figure 4, B). Despite leukocytes representing a minor fraction of blood cells (about thousand times less cells compared to RBC and about fifty times less than platelets), we observed that the affinity of Dd in blood is highly in favor of leukocytes, independently of their subtypes.

\section{Dd uptake by drug-resistant cancer cells}

Dd, similarly to adenoviruses, attaches to cell membrane and penetrates cells by receptormediated endocytosis, therefore we probed if it would be internalized by drug-resistant cells, 
overexpressing P-glycoproteins, drug efflux pumps for xenobiotic compounds with broad substrate specificity. For this purpose we had chosen human uterus sarcoma cell line MES-SA and its multidrug-resistant counterpart, MES-SA Dx5. Since, to the best of our knowledge, the expression of Dd receptors on human uterus sarcoma cells was not reported hitherto, we started from the verification of their presence. The fluorescence microscopy imaging with the use of specific antibodies confirmed the presence of $\alpha v \beta 3$ and $\alpha v \beta 5$ integrins, which are involved in Dd internalization, on the surface of MES-SA and MES-SA Dx5 cells (Figure 5, A). The confocal microscopy analysis, performed after 2 hour incubation of MES-SA and MES-SA Dx5 cells with $10 \mu \mathrm{g}$ of Dd, demonstrated lack of differences in the amount and intracellular localization of the vector (Figure 5, $B$ and $C$ ). While analyzing Dd penetration, we observed that particle endocytosis increased up to $2 \mathrm{~h}$ (Figure $5, B$ and $C$ ), while the amount of intracellular Dd diminished at later time points (data not shown).

\section{Doxorubicin conjugation to Dd particle}

We wanted to verify if conjugation of doxorubicin to Dd (Dd-DOX) improves antibiotic delivery to drug-resistant cancer cells. In the preparation of Dd-DOX conjugates we used the NHS-ester-activated adipic acid as a crosslinker for coupling lysine residues of Dd with the primary amine groups of doxorubicin. Doxorubicin concentration in Dd-DOX conjugates, determined by fluorescence intensity measurement with the standard curve for DOX and UVvis spectra of DOX and Dd-DOX, was $3 \mu \mathrm{M}$. The average Dd loading with drug was estimated to about 90 molecules of DOX per one molecule of vector.

\section{Intracellular distribution of Dd-DOX and free DOX}

Cell entry of Dd-DOX conjugate (and free DOX) was monitored by confocal microscopy. Figure 6 shows subcellular localization of free DOX and Dd-DOX in MES-SA and MES-SA Dx5 cell lines four hours after application. The treatment with free DOX resulted in the classical image of nuclear drug localization in MES-SA cells, whereas only a negligible 
fluorescence of DOX (green) can be observed inside MES-SA Dx5 cells, which is most likely related to the expression of P-gp protein in these cells. ${ }^{17}$ All MES-SA and MES-SA DX5 cells were found to be transduced with Dd-DOX, as indicated by the presence of Dd (red stain) in cytoplasm of each cell. In both cell lines similar intracellular localization was observed for DOX, delivered in the form of conjugate, with DOX fluorescence signal present in the nuclei as well as in the cytoplasm of transduced cells. The presence of DOX molecules distributed in cytoplasm suggests that they were still attached to the carrier, while the majority of DOX was already released from conjugate and delivered into the nucleus.

\section{Cytotoxicity of Dd-DOX conjugate}

According to our results of MTT assay, the application of the vector alone did not affect the viability of either MES-SA or MES-SA Dx5 cells (Figure 7, A), however, delivery of DdDOX conjugate resulted in diminished, concentration-dependent viability of both, drugresistant and drug-sensitive cells after incubation for 48 hours. After treatment with equal concentrations of doxorubicin in a form of conjugate in comparison with a free form of drug, the $\mathrm{IC}_{50}$ (half maximal inhibitory concentration) value, according to MTT assays, was on average fourfold lower for Dd-DOX conjugate than that for free DOX for MES-SA, and approximately five fold lower for MES-SA Dx5 cells (Figure 7, B, Table 1).

\section{Dd storage conditions}

For the further therapeutic uses of Dd the elaboration of method for convenient particle storage and transportation was needed. We proposed simple protocol for Dd lyophilization for prolonged storage. Electron microscopy and In-cell ELISA analyses of water-reconstructed samples indicated that Dd purified in two-step protocol based on size exclusion and anion exchange chromatography ${ }^{18}$ can be frozen and lyophilized in the presence of sodium chloride and cryoprotectants (sucrose and mannitol), without inducing aggregation, losing structural integrity or penetration capabilities (Figure 8). Of note, even preparations lyophilized in the 
absence of cryoprotectants after reconstitution in water still contained a large proportion of well-formed dodecahedra, although a significant amount of free pentameric bases was observed (data not shown). Lyophilized Dd can be stored at temperatures up to $37{ }^{\circ} \mathrm{C}$, which means that protein could be transported and used without refrigeration (Figure 8, A). Moreover, we observed no substantial difference in cell penetration capabilities before and after Dd lyophilization and storage at indicated temperatures (Figure 8, B). Clearly, the presence of cryoprotectants did not affect cell internalization.

\section{Discussion}

Our aim was the evaluation of adenoviral dodecahedron tropism and identification of VLP properties important for its development as drug delivery platform. Selection of suitable route of administration is essential for accurate planning of dodecahedron application in cancer treatment. The low tumor uptake, observed upon intravenous injection, suggests limited usefulness of this route of administration for Dd-mediated delivery to melanoma tumor. Our previous studies showed that when applied intraperitoneally, Dd conjugates carrying anticancer agents efficiently targeted tumors in orthotopic hepatocellular carcinoma rat model. ${ }^{14}$ After intraperitoneal injection Dd conjugates entered into the mesenteric blood circulation and then were carried via the portal vein directly to liver finally accumulating in HCC tumor but not in healthy tissues. ${ }^{14}$ This discrepancy may be explained by differences in the angiogenesis and permeability of blood vessels between xenograft model used for intravenous administration and allograft model used for intraperitoneal injection. ${ }^{14}$ As opposed to intravenous administration, the intratumoral injection resulted in long-lasting persistence of the vector in melanoma tumor without spreading in off-target organs. Our results suggest that intratumoral application may decrease eventual drug cytotoxicity in healthy tissues, enabling usage of higher concentrations of Dd-drug conjugates. To conclude, our previous and recent results, we consider Dd suitability for local application. 
Surface functionalization of nanoparticles with specific ligands enables not only specific cellular targeting but also promotes crossing biological barriers such as a BBB. ${ }^{19}$ It is a common approach, for example in the case of Cowpea mosaic virus (CPMV) VLPs conjugation with antibodies against Human Epithelial Receptor- $2^{20}$ or with transferrin, ${ }^{21}$ which was also used for functionalization of zolendronic acid (ZOL)NPs. ${ }^{22}$ The improved Dd targeting of tumor upon intravenous application may be achieved by particle modification, for example with short homing peptides, which specifically recognize and bind to surface molecules on tumor cells or tumor vasculature. ${ }^{23}$ It is worth mentioning, that Dd structural modification has already been validated for insertion of antigen peptides into external particle positions without disrupting its integrity, nor penetration properties. ${ }^{15}$ Observed Dd lack of ability to pass through the BBB excludes the possibility of using non-targeted dodecahedron as a vector for factors acting on the central nervous system (CNS), thereby enabling the safe therapeutic applications of the particle, without possible adverse side effects on CNS.

Dd distribution through the mice body resulted in the preferential accumulation of VLP in animal skin. The interaction with HSPGs present at the surface of cells and in the extracellular matrix of skin may be involved in Dd affinity to this organ, while similar tropism was not observed for the $\mathrm{Ad} 3 ;^{24}$ it is worth to emphasize that Ad3 does not use HSPGs as receptors for internalization. Together, Dd tropism to animal skin observed upon i.v. application and ability for efficient penetration of dermal fibroblasts observed in vitro suggests the utility of VLP as a carrier for delivery to skin dermis. Importantly, the ability to penetrate primary cells, first shown for the primary human astrocytes ${ }^{11}$, is an important attribute of the vector for its potential application, since primary cells are difficult to transfect using electroporation or chemical transfection reagents. ${ }^{25}$ Thus, the use of Dd offers a new solution to this challenge. 
Previously reported results, on Dd stability in human serum, ${ }^{13}$ prompted us to investigate carrier affinity to blood cells. Interactions with blood cells may have important consequences for undesirable vector elimination from the blood stream and its degradation, as it was demonstrated for recombinant adenovirus type 5 (Ad5) gene therapy vectors. ${ }^{26}{ }^{27}$ It was also shown for Ad3 that surface interaction with platelets and granulocytes is mediated by binding to desmoglein 2 (DSG-2, the receptor of adenovirus fiber), ${ }^{28}$ which, however, cannot function as the attachment receptor for fiber-less dodecahedra used in our studies. Importantly, known receptors essential for Dd internalization, are present on the surface of freshly isolated circulating leukocytes in low amounts. ${ }^{29}$ It seems that cell surface molecules, other than already identified receptors, may be involved in WBC recognition since the low level of $\alpha v \beta 3$ integrins and HSPGs expressed by small fraction of cells might be not sufficient to support efficient penetration of about $85 \%$ leukocytes demonstrated in current study. It is worthwhile to mention that $\mathrm{Ad} 2$ attachment to and infection of monocytic cells is facilitated by the described interaction of penton base protein with $\alpha_{M} \beta 2$ integrin. ${ }^{30}$ Within $\alpha_{M} \beta 2$ integrin, the $\alpha \mathrm{MI}$-domain is responsible for the ability of multiligand binding. Based on the algorithm that predicts the $\alpha$ MI-domain binding sites in the $\alpha_{M} \beta 2$ ligands ${ }^{31}$ and analysis of $\mathrm{Ad} 3 \mathrm{~Pb}$ sequence and structure ${ }^{32}$ we predict that the relevant recognition motif is localized in the region of externally exposed variable loop of $\mathrm{Ad} 3 \mathrm{~Pb}$. Mechanistic studies on the uptake of Dd in human leukocytes could deepen the understanding of this ability. However, the most important point is the distinct Dd transduction efficiency for white blood cells, since it could lead to the use of this vector for the transport of active molecules targeting leukocytes, for example as a putative drug carrier targeting leukaemia cells, in particular for in vitro testing of potential anticancer agents intended to treat leukocyte disorders. The suitability of Dd for such application is supported by the lack of cytotoxicity of this VLP in human peripheral blood mononuclear cells. ${ }^{15}$ 
A major problem facing current cancer treatment is tumor resistance to chemotherapy. The most common mechanism of resistance is mediated through the drug efflux pump, P-gp 1 protein, that expels membrane-embedded exogenous molecules. ${ }^{33}$ Various delivery systems based on mesoporous silica NPs,${ }^{34}$ liposomes ${ }^{35,36}$ and micelles ${ }^{37-38}$ have been developed to circumvent drug resistance of cancer cells. Our approach is based on the remarkable cell penetration capability of Dd nanoparticles. While free doxorubicin enters cell by a passive diffusion across the plasma membrane ${ }^{39}$ and it may be expelled by Pgp-1 from drug-resistant cells, ${ }^{40}$ Dd-DOX conjugate penetrates cells via receptor-mediated endocytosis, which conceivably does not allow doxorubicin recognition by the efflux pumps that are localized in the plasma membrane. ${ }^{41}$ Confocal microscopy images of uterine sarcoma MES-SA cells treated with free DOX are consistent with literature data on rapid intercalation of DOX within nuclear $\mathrm{DNA}^{42}$ in sensitive cells and significantly reduced nuclear uptake in case of cells expressing P-gp efflux pumps. Treatment of MES-SA and MES-SA Dx5 drug-resistant cells with Dd-DOX resulted in the high nuclear and, rather low, cytoplasmic localization of DOX carried by Dd (Figure 6). DOX fluorescence in cytoplasm is associated with drug conjugation to Dd whereas DOX accumulation in the nucleus must be due to its release from conjugate, because Dd does not enter nucleus ${ }^{10}$. The nuclear accumulation of DOX 4 hours post application of conjugate suggests rapid proteolysis of the vector, which might result in liberating of peptides containing DOX, probably able to easily translocate to the nucleus. Indeed, the dodecahedric vector was observed to undergo rapid proteolysis inside transduced cells. ${ }^{13}$ Consistently with the enhanced drug delivery to MES-SA and MES-SA Dx5 cells, Dd-DOX represents higher cytotoxicity than free DOX in both cell lines, with more pronounced effect exerted against drug resistant cells (Figure 6, Table 1). The current results are in agreement with our previous in vitro studies, when we observed efficient delivery of Dd-DOX conjugate to human HeLa and rat Morris hepatoma MH-3924A cells, with the 
similar pattern of DOX intracellular localization. ${ }^{14}$ Reported previously cell growth inhibition upon Dd-DOX treatment of drug sensitive HeLa cells and two liver cancer cell lines: rat Morris hepatoma MH-3924A and human hepatocellular carcinoma PLC/PRF/5, was achieved with 5 fold lower amount of doxorubicin in conjugate, when compared to application of free drug. These data are consistent with presented here results of cytotoxicity tests performed on uterine sarcoma cell lines. On the grounds of our results we consider Ad3 Dd to be a promising vector for intracellular delivery that enables to overcome drug-resistance in cancer cells.

Additionally this paper contains a description of simple and useful procedure for the vector long-term storage at broad spectrum of temperatures, without disrupting vector structure and properties. Thus we believe that Dd meets the important requirement for drug carriers, which is convenient storage and transportation. 


\section{Figure captions}

Figure 1. Dd-AF680 localization after systemic application. Nude mice were injected intravenously with $33 \mu \mathrm{M}(200 \mu \mathrm{l}) \mathrm{Dd}-\mathrm{AF} 680$. A) In vivo fluorescence imaging of Dd-AF680 at T0 (before injection), at $5 \mathrm{~h}$ and $24 \mathrm{~h}$ post injection (p.i.). T - tumor, LN - lymph nodes, $\mathrm{p} / \mathrm{sec} / \mathrm{cm}^{2}$ - number of photons per second, per square centimeter. B) Ex vivo fluorescence imaging of isolated organs and tumors 5 and 24 h p.i. C) Ex vivo fluorescence analysis of isolated organs and tumors for Dd-AF680 injected and control (Ctl, non-injected) mice, at indicated times p.i.

Figure 2. Dd-AF680 localization after intratumoral injection. Nude mice were injected with $8.25 \mu \mathrm{M}(50 \mu \mathrm{l})$ Dd-AF680. A) In vivo fluorescence imaging of Dd-AF680 at T0 (before injection), $5 \mathrm{~h}$ and $24 \mathrm{~h}$ post injection (p.i.). $\mathrm{T}$ - tumor, $\mathrm{p} / \mathrm{sec} / \mathrm{cm}^{2}$ - number of photons per second, per square centimeter. B) Ex vivo fluorescence imaging of isolated organs and tumors 5 and 24 hp.i. C) Ex vivo fluorescence analysis of isolated organs and tumors for Dd-AF680 injected and control (Ctl, non-injected) mice at indicated times p.i.

Figure 3. Flow cytometry analysis of Dd uptake in primary dermal fibroblasts. Fibroblasts were incubated with 10 or $30 \mu \mathrm{g} / \mathrm{ml}$ of Dd-AF647 respectively for $1 \mathrm{~h}$ at $37^{\circ} \mathrm{C}$. A) A bar graph representing mean values and standard deviations of summarized data from three separate set of experiments and triplicate measurements per each experiment. B) A representative analysis for one experiment. On histograms, the percentage of cells transduced by Dd-AF647 is reported for each population as well as mean fluorescence intensity (MFI) at increased concentration.

Figure 4. Blood cells penetration. Fresh blood cells were exposed to Dd-AF647 for $1 \mathrm{~h}$ at 37 ${ }^{\circ} \mathrm{C}, 0.7$ up 12.2 pmoles, and analyzed by flow cytometry. Platelets, red blood cells (RBC) and leukocytes were gated by their respective specific expression of CD41a, CD231a and CD45. The threshold percent gate was set-up by cell type for background on samples in the absence of Dd treatment. A) A representative analysis on a blood sample from one donor. On histograms, the percentage of cells transduced by Dd-AF647 is reported for each population as well as mean fluorescence intensity (MFI) at increased concentration. B) The analysis of detailed subpopulations of leukocytes is shown: T-cells are gated on CD3 expression, myeloid are $\mathrm{CD}_{3} 3^{+}$cells and $\mathrm{CD} 14^{+}$cells are mainly monocytes. C) Data from four distinct donors are shown and summarized by a histogram of MFI measured on blood cells. Cell counts are 
indicated in the tables (times $10^{9}$ cells/l). On histograms, the percentage of cells transduced by Dd-AF647 is reported for each population as well as MFI at increased concentration. Data represent mean values with standard deviations for four experiments and triplicate measurements for each experiment. Data were compared by applying Student's $t$-test, which indicated statistically significant differences between leukocytes compared to RBC and platelets for all amounts of Dd from 1.4 up to 12.2 pmoles (** $\mathrm{P}<0.01$ ). For 0.7 pmoles of Dd the P value was lower than 0.05 .

Figure 5. Dd penetration of drug-resistant cells. A) Fluorescence imaging of integrins $\alpha v \beta 3$ and $\alpha v \beta 5$ in MES-SA and MES-SA Dx5 cells. Integrins $\alpha v \beta 3$ and $\alpha v \beta 5$ were detected with anti- $\alpha v \beta 3$ or anti- $\alpha v \beta 5$, respectively and secondary Alexa Fluor 488 conjugated antibody (green), cell nuclei were stained with DAPI. Scale bar - $10 \mu \mathrm{m}$. B) Confocal microscope images of Dd internalization to MES-SA and MES-SA Dx5 cells. Dd entry kinetics was measured at indicated time points, between 0 - $120 \mathrm{~min}$. Intracellular Dd was recognized with rabbit polyclonal anti-Dd serum and secondary anti-rabbit antibody conjugated with Alexa Fluor 488 (green), cell nuclei were stained with DAPI (blue). Scale bar - $10 \mu \mathrm{m}$. C) Corrected total cell fluorescence (CTCF) measurement for MES-SA and MES-SA Dx5 cells related to indicated times of incubation with Dd. Data represent mean \pm s.d. for five measurements.

Figure 6. Intracellular uptake of Dd-DOX and free DOX. MES-SA and MES-SA Dx5 cells were incubated with $2 \mu \mathrm{M}$ Dd-DOX conjugate or free DOX for 48 hours, respectively. Intracellular Dd was recognized with rabbit polyclonal anti-Dd serum and secondary antirabbit antibody conjugated with Alexa Fluor 647 (AF647). Images were obtained with Zeiss Axio Observer Z1 inverted microscope. The confocal microscope settings were the same between specimens. DOX and AF647 fluorescence was recorded with software-added green and red pseudo color, respectively, using Fiji software.

Figure 7. Cytotoxic effect of Dd-DOX conjugate determined by MTT assay. A) MES-SA and MES-SA Dx 5 cells were treated with Dd (6 $\mu \mathrm{g})$, Dd-DOX (2, 4, $6 \mu \mathrm{g}$ delivering 50, 100, 150 $\mathrm{nM}$ DOX respectively) and free DOX (50, 100, $150 \mathrm{nM})$. Cell viabilities were compared to their respective non-treated controls. Data were compared by applying Student's $t$-test, which indicated statistically significant differences between DOX and Dd-DOX treated cells $(* \mathrm{P}<$ $0.05, * * \mathrm{P}<0.01)$. Data represent mean \pm s.d. $(\mathrm{n}=3$ experiments, five wells per treatment $)$. B) Dose-response curves for Dd-DOX conjugates and free DOX for the MES-SA (left panel) and MES-SA Dx5 (right panel) cell lines. Various concentrations of Dd-DOX (20, 50, 100, 
150, 260, $390 \mathrm{nM})$ and $\operatorname{DOX}(50,100,150,300,450,600 \mathrm{nM})$ were applied, and the viability of cells was determined by the MTT assay. Non-linear regression analysis was performed using Origin software (OriginLab Corporation, USA). The averaged percentage corresponding to viability of cells was plotted against the logarithm of the concentration of Dd-DOX conjugates and free DOX. Curves were fitted to a dose-response function according to the equation $\mathrm{y}=\mathrm{A} 1+\left((\mathrm{A} 2-\mathrm{A} 1) /\left(1+10^{\wedge}(\log \mathrm{x} 0-\mathrm{x}) \bullet \mathrm{p}\right)\right)$. Each value is the mean \pm standard error obtained for three experiments ( $n=3$ experiments, five wells per treatment).

Figure 8. Dd lyophilization and storage in $20 \mathrm{mM}$ HEPES buffer containing $0.1 \mathrm{mM}$ EDTA, $150 \mathrm{mM} \mathrm{NaCl}$ and cryoprotectants (sucrose $0.4 \% \mathrm{v} / \mathrm{v}$ and mannitol $0.4 \% \mathrm{v} / \mathrm{v}$ ). Separate 300 $\mu \mathrm{g}$ aliquots were lyophilized and stored for 10 days at indicated temperatures and reconstituted in sterile water. A) Electron microscopy analysis. Upper panel, on the left: control sample before lyophilization (Dd), on the right: sample after freezing in liquid nitrogen, stored for 10 days at $-20{ }^{\circ} \mathrm{C}$ (control freezing). Lower panel - samples after lyophilization stored at indicated temperatures. Scale bar $100 \mathrm{~nm}$. B) In-cell ELISA analysis of reconstituted Dd internalization to HeLa cells. Cells were incubated for $2 \mathrm{~h}$ at $37{ }^{\circ} \mathrm{C}$ with 4 $\mu \mathrm{g}$ of Dd. Data represent mean \pm s.d. for triplicates.

\section{Table 1}

Concentration inhibiting $50 \%$ of cell growth $\left(\mathrm{IC}_{50}\right)$ reached after $48 \mathrm{~h}$ of treatment with DdDOX and free DOX in MES-SA and MES-SA Dx5 cells. Data represent mean values and standard deviations (SD) for three separate experiments. 


\section{Acknowledgements}

We are grateful to M. Lichocka (IBB, Warsaw, Poland) for help with confocal microscopy and M. Mielecki (IBB, Warsaw, Poland) for help with statistical analysis.

\section{Author Contributions}

Designed the experiments: J.C., M.J., D.L., E.S. Performed the experiments: P.D., M.J., D.L., M.P.B., E.S., I. S. Analyzed the data: J.C., P.D., M.J., D.L., E.S. Contributed reagents/materials/analysis tools: J.C., D.L., E.S. Wrote the paper: J.C., M.J., D.L., E.S. 


\section{References}

1. Anselmo AC, Mitragotri S. Nanoparticles in the clinic. Bioeng Transl Med 2016;1:1029.

2. Gordon AN, Fleagle JT, Guthrie D, Parkin DE, Gore ME, Lacave AJ. Recurrent epithelial ovarian carcinoma: a randomized phase III study of pegylated liposomal doxorubicin versus topotecan. J. Clin. Oncol 2001;19:3312-22.

3. Gradishar WJ, Tjulandin S, Davidson N, Shaw H, Desai N, Bhar P, et al. Phase III trial of nanoparticle albumin-bound paclitaxel compared with polyethylated castor oil-based paclitaxel in women with breast cancer. J. Clin. Oncol 2005;23:7794-803.

4. Rahovie MJ, Nagasawa M, Swartz JR. Virus-like particles: next generation nanoparticles for therapeutic delivery. Bioenginiring \& Translational Medicine 2017;2:43-57.

5. Wickham TJ, Mathias, Cheresh DA, Nemerow GR. Integrins alpha $v$ beta 3 and alpha $v$ beta 5 promote adenovirus internalization but not virus attachment. Cell 1993;73:30919.

6. Vivès RR, Lortat-Jacob H, Chroboczek J, Fender P. Heparan sulfate proteoglycan mediates the selective attachment and internalization of serotype 3 human adenovirus dodecahedron. Virology 2004;321:332-40.

7. Fender P, Schoehn G, Perron-Sierra F, Tucker GC, Lortat-Jacob H. Adenovirus dodecahedron cell attachment and entry are mediated by heparan sulfate and integrins and vary along the cell cycle. Virology 2008;371:155-64.

8. Eliceiri BP, Cheresh DA. The role of alpha $\mathrm{v}$ integrins during angiogenesis: insights into potential mechanisms of action and clinical development. $J$ Clin Invest 1999;103:1227-30.

9. Pasqualini R, Koivunen E, Ruoslahti E. Alpha $\mathrm{v}$ integrins as receptors for tumor 
targeting by circulating ligands. Nat Biotechnol 1997;15:542-6.

10. Fender P, Ruigrok RW, Gout E, Buffet S, Chroboczek J. Adenovirus dodecahedron, a new vector for human gene transfer. Nat Biotechnol 1997;15:52-6.

11. Garcel A, Gout E, Timmins J, Chroboczek J, Fender P. Protein transduction into human cells by adenovirus dodecahedron using WW domains as universal adaptors. $J$ Gene Med 2006;8:524-31.

12. Sumarheni S, Gallet B, Fender P. The Use of Adenovirus Dodecahedron in the Delivery of an Enzymatic Activity in the Cell. Biotechnol Res Int 2016;2016:5030589.

13. Zochowska M, Paca A, Schoehn G, Andrieu JP, Chroboczek J, Dublet B, et al. Adenovirus dodecahedron, as a drug delivery vector. PLoS One 2009;4:e5569.

14. Zochowska M, Piguet AC, Jemielity J, Kowalska J, Szolajska , Dufour JF, et al. Viruslike particle-mediated intracellular delivery of mRNA cap analog with in vivo activity against hepatocellular carcinoma. Nanomedicine: Nanotechnology, Biology, and Medicine 2015;11:67-76.

15. Szurgot I, Szolajska E, Laurin D, Lambrecht B, Chaperot L, Schoehn G, et al. Selfadjuvanting influenza candidate vaccine presenting epitopes for cell-mediated immunity on a proteinaceous multivalent nanoplatform. Vaccine 2013;31:4338-46.

16. Simon-Santamaria J, Rinaldo $\mathrm{CH}$, Kardas $\mathrm{P}$, Malovic R, Li I, Elvevold K, et al. Efficient Uptake of Blood-Borne BK and JC Polyomavirus-Like Particles in Endothelial Cells of Liver Sinusoids and Renal Vasa Recta. Plos One 2014;9:e111762.

17. Wesolowska O,Paprocka M, Kozlak J, Motohashi N, Dus D, Michalak K. Human sarcoma cell lines MES-SA and MES-SA/Dx5 as a model for multidrug resistance modulators screening. Anticancer Res 2005;25:383-9.

18. Szurgot I, Jedynak M, Podsiadla-Bialoskorska M, Piwowarski J, Szolajska E, Chroboczek J. Adenovirus Dodecahedron, a VLP, Can be Purified by Size Exclusion Chromatography Instead of Time-Consuming Sucrose Density Gradient 
Centrifugation. Mol Biotechnol 2015;57:565-73.

19. Caraglia M, De Rosa G, Salzano G, Santini D, Lamberti M, Sperlongano P, et al. Nanotech revolution for the anti-cancer drug delivery through blood-brain barrier. Curr Cancer Drug Targets 2012;12:186-96.

20. Chatterji A, Ochoa W, Shamieh L, Salakian SP, Wong SM, Clinton G. Chemical conjugation of heterologous proteins on the surface of Cowpea mosaic virus. Bioconjug Chem 2004;15:807-13.

21. Sen Gupta S, Kuzelka J, Singh P, Lewis WG, Manchester M, Finn MG. Accelerated bioorthogonal conjugation: a practical method for the ligation of diverse functional molecules to a polyvalent virus scaffold. Bioconjug Chem 2005;16:1572-9.

22. Porru M, Zappavigna S, Salzano G, Luce A, Stoppacciaro A, Balestrieri ML, et al. Medical treatment of orthotopic glioblastoma with transferrin conjugated nanoparticles encapsulating zoledronic acid. Oncotarget 2014;5:10446-59.

23. Kapoor P, Singh H, Gautam A, Chaudhary K, Kumar R, Raghava GP. TumorHoPe: A Database of Tumor Homing Peptides. Plos One 2012;7:e35187.

24. Wang H, Beyer I, Persson J, Song H, Li ZY, Richter M, et al. A new human DSG2transgenic mouse model for studying the tropism and pathology of human adenoviruses. $J$ Virol 2012;86:6286-302.

25. Hunt MA, Currie MJ, Robinson BA, Dachs GU. Optimizing transfection of primary human umbilical vein endothelial cells using commercially available chemical transfection reagents. J Biomol Tech 2010;21:66-72.

26. Stone D, Liu Y, Shayakhmetov D, Li ZY, Ni S, Lieber A. Adenovirus-Platelet Interaction in Blood Causes Virus Sequestration to the Reticuloendothelial System of the Liver. $J$ Virol 2007;81:4866-71.

27. Lyons M, Onion D, Green NK, Aslan K, Rajaratnam R, Bazan-Peregrino M, et al. 
Adenovirus type 5 interactions with human blood cells may compromise systemic delivery. Mol Ther 2006;14:118-28.

28. Wang H, Li ZY., Liu Y, Persson J, Beyer I, Möller T, et al. Desmoglein 2 is a receptor for adenovirus serotypes 3, 7, 11 and 14. Nat Med 2011;17:96-104.

29. Huang S, Endo RI, Nemerow GR. Upregulation of integrins alpha vbeta 3 and alpha v beta 5 on human monocytes and T lymphocytes facilitates adenovirus-mediated gene delivery. J. Virol 1995;69: 2257-63.

30. HuangS. KamataT, TakadaY, Ruggeri, ZM, Nemerow GR. Adenovirus interaction with distinct integrins mediates separate events in cell entry and gene delivery to hematopoietic cells. J Virol 1996;70:4502-28.

31. Podolnikova NP, Podolnikov AV, Haas TA, Lishko VK, Ugarova TP. Ligand recognition specificity of leukocyte integrin $\alpha \mathrm{M} \beta 2$ (Mac-1, CD11b/CD18) and its functional consequences. Biochemistry 2015;54:1408-20.

32. Szolajska E, Burmeister WP, Zochowska M, Nerlo B, Andreev I, Schoehn G, et al. The structural basis for the integrity of adenovirus Ad3 dodecahedron. J. PLoS One 2012;7:e46075.

33. Krishna R, Mayer LD. Multidrug resistance (MDR) in cancer. Mechanisms, reversal using modulators of MDR and the role of MDR modulators in influencing the pharmacokinetics of anticancer drugs. Eur J Pharm Sci 2000;11:265-83.

34. Shen J, He Q, Gao Y, Shi J, Li Y. Mesoporous silica nanoparticles loading doxorubicin reverse multidrug resistance: performance and mechanism. Nanoscale 2011;3:4314-22.

35. Kang D, Kang HK, Gwak HS, Han HK, Lim SJ. Liposome composition is important for retention of liposomal rhodamine in P-glycoprotein-overexpressing cancer cells. Drug Deliv 2009;16:261-7. 
36. Perillo E, Porto S, Falanga A, Zappavigna S, Stiuso P, Tirino V, et al. Liposome armed with herpes virus-derived gH625 peptide to overcome doxorubicin resistance in lung adenocarcinoma cell lines. Oncotarget 2016;7:4077-92.

37. Lee ES, Na K, Bae YH.Doxorubicin loaded $\mathrm{pH}$-sensitive polymeric micelles for reversal of resistant MCF-7 tumor. J Control Release 2005;103:405-18.

38. Mohajer G, Lee ES, Bae YH. Enhanced intercellular retention activity of novel pHsensitive polymeric micelles in wild and multidrug resistant MCF-7 cells. Pharm Res 2007;24:1618-27.

39. Frezard F, Garnier-Suillerot A. Comparison of the membrane transport of anthracycline derivatives in drug-resistant and drug-sensitive K562 cells. Eur $J$ Biochem 1991;196:483-91.

40. Harker WG, Sikic BI. Multidrug (pleiotropic) resistance in doxorubicin-selected variants of the human sarcoma cell line MES-SA. Cancer Res 1985;45:4091-6.

41. Sharom FJ. Complex Interplay between the P-Glycoprotein Multidrug Efflux Pump and the Membrane: Its Role in Modulating Protein Function. Front Oncol 2014;4:41.

42. Belloc F, Lacombe F, Dumain P, Lopez F, Bernard P, Boisseau MR, et al. Intercalation of anthracyclines into living cell DNA analyzed by flow cytometry. Cytometry 1992;13:880-5. 